\title{
The Three-point Correlation Function of Galaxies Determined from the Las Campanas Redshift Survey
}

\author{
Y.P. Jing ${ }^{1}$, G. Börner ${ }^{1,2}$ \\ ${ }^{1}$ Research Center for the Early Universe, School of Science, University of Tokyo, \\ Bunkyo-ku, Tokyo 113, Japan \\ ${ }^{2}$ Max-Planck-Institut für Astrophysik, Karl-Schwarzschild-Strasse 1, 85748 Garching, \\ Germany \\ e-mail: jing@utaphp2.phys.s.u-tokyo.ac.jp, grb@mpa-garching.mpg.de
}

\begin{abstract}
We report the measurement of the three-point correlation function (3PCF) of galaxies for the Las Campanas Redshift Survey (LCRS). We have not only measured the $3 \mathrm{PCF}$ in redshift space but also developed a method to measure the projected $3 \mathrm{PCF}$ which has simple relations to the real space $3 \mathrm{PCF}$. Both quantities have been measured as a function of triangle size and shape with only a fractional uncertainty in each individual bin. Various tests derived from mock catalogs have been carried out to assure that the measurement is stable and that the errors are estimated reliably. Our results indicate that the $3 \mathrm{PCF}$ both in redshift space and in real space have small but significant deviations from the well-known hierarchical form. The $3 \mathrm{PCF}$ in redshift space can be fitted by $Q_{r e d}(s, u, v)=0.5 \cdot 10^{\left[0.2+0.1\left(\frac{s}{s+1}\right)^{2}\right] v^{2}}$ for $0.8<s_{12}<8 h^{-1} \mathrm{Mpc}$ and $s_{31}<16 h^{-1} \mathrm{Mpc}$, and the projected 3PCF by $Q_{\text {proj }}\left(r_{p}, u, v\right)=0.7 r_{p}^{-0.3}$ for $0.2<r_{p 12}<3 h^{-1} \mathrm{Mpc}$ and $r_{p 31}<6 h^{-1} \mathrm{Mpc}\left(s\right.$ and $r_{p}$ are in unit of $h^{-1} \mathrm{Mpc}$ ), though a systematic weak increase of $Q_{p r o j}\left(r_{p}, u, v\right)$ with $v$ at $r_{p}>1 h^{-1} \mathrm{Mpc}$ is noted. The real-space $Q(r, u, v)$ for $0.2 \lesssim r_{12} \lesssim 3 h^{-1} \mathrm{Mpc}$ and $r_{31} \lesssim 6 h^{-1} \mathrm{Mpc}$ can be well described by half the mean $3 \mathrm{PCF}$ predicted by a CDM model with $\Omega_{0} h=0.2$.

The general dependence of the $3 \mathrm{PCF}$ on triangle shape and size is in qualitative agreement with the CDM cosmogonic models. Quantitatively the 3PCF of the models may depend on the biasing parameter and the shape of the power spectrum, in addition to other model parameters. Taking our result together with the constraints imposed by the two-point correlation function and the pairwise velocity dispersion of galaxies also obtained from the LCRS, we find that we have difficulties to produce a simple model that meets all constraints perfectly. Among the CDM models considered, a flat model with $\Omega=0.2$ meets the $2 \mathrm{PCF}$ and PVD constraints, but gives higher values for the $3 \mathrm{PCF}$ than
\end{abstract}


observed. This may indicate that more sophisticated bias models or a more sophisticated combination of model parameters must be considered.

Subject headings: galaxies: clustering - galaxies: distances and redshifts large-scale structure of Universe - cosmology: theory - dark matter

\section{Introduction}

Correlation functions are very powerful statistics to describe the large scale structures in the Universe (Peebles 1980, hereafter P80). The lowest order, the two-point correlation function $(2 \mathrm{PCF}) \xi(r)$, has been widely used to measure the clustering strength of galaxies and to confront models of cosmic structure formation. Quite a number of large galaxy catalogs, both angular and redshift, have been used to determine the $2 \mathrm{PCF}$, and this statistic has now been established quite well (Jing, Mo, \& Börner 1998, hereafter JMB98; Lin et al. 1998 in preparation; Lin et al. 1997; Tucker et al. 1997; Ratcliffe et al. 1997; Baugh 1996; Hermit et al. 1996; Strauss \& Willick 1995 for a review before 1995). This statistic has produced several constraints on theoretical models already despite the fact that there are many ingredients to a specific model which can be optimally adapted to the properties of the galaxy sample. The cosmological parameters, like the initial power spectrum of the DM component and the bias, i.e. the difference in the clustering of galaxies and DM particles, can all be adjusted to some extent.

The three-point correlation function (3PCF) $\zeta\left(r_{12}, r_{23}, r_{31}\right)$ is a further statistic useful in characterizing the clustering of galaxies (P80). Its measurement can give additional constraints for cosmogonic models. The determination of the 3PCF was pioneered by Peebles and his coworkers in the seventies. Based on their careful analysis of the Lick and Zwicky angular catalogs of galaxies they propose a so-called "hierarchical" form

$$
\zeta\left(r_{12}, r_{23}, r_{31}\right)=Q\left[\xi\left(r_{12}\right) \xi\left(r_{23}\right)+\xi\left(r_{23}\right) \xi\left(r_{31}\right)+\xi\left(r_{31}\right) \xi\left(r_{12}\right)\right]
$$

with the constant $Q \approx 1.29 \pm 0.2$. This form is valid for scales $r \lesssim 3 h^{-1} \mathrm{Mpc}(\mathrm{P} 80)$. The analysis of the ESO-Uppsala catalog of galaxies (Lauberts 1982) by Jing, Mo, \& Börner (1991) supports this result. The 3PCF was also examined for the CfA, AAT and KOSS redshift samples of galaxies (Peebles 1981; Bean et al. 1983; Efstathiou \& Jedrzejewski 1984; Hale-Sutton et al. 1989). Because all these redshift samples are small (with $<2000$ galaxies), these authors were not able to examine the validity of the hierarchical form in redshift space. Instead they just forced a fit of the hierarchical form and obtained the value of $Q$. The $Q$ value of redshift samples obtained in this way is around 0.6 (Efstathiou \& 
Jedrzejewski 1984), much smaller than the value advocated by Peebles and his coworkers. The difference may partially be attributed to the redshift distortion effect which reduces the $Q$ value (Matsubara 1994). The skewness analysis of the 1.2 Jy IRAS survey of galaxies has given a similar $Q$ value (Bouchet et al. 1993).

The hierarchical form (Eq. 11) is purely empirical. There is no solid theoretical argument supporting this form. In contrast, the second-order perturbation theory predicts that $Q$ depends on the shape of the triangle and on the slope of the linear power spectrum (Fry 1984) in the linear regime. For a CDM-like power spectrum with a slope which changes with the scale, $Q$ then varies with the size and shape of a triangle (Jing \& Börner 1997). Even in the strongly non-linear regime where the hierarchical form was established, the CDM models do not seem to obey this form as demonstrated by Matsubara \& Sutg (1994) based on N-body simulations. Recently, Yano \& Gouda (1997) have re-examined, based on the BBGKY equation, the stable clustering (strongly non-linear) problem and pointed out that the hierarchical form holds if the clustering is stable. The question is if the condition of stable clustering can be achieved in the real Universe (Jain 1997).

The 3PCF of galaxies carries much useful information which is important for cosmogonic models. The theories based on CDM models predict that the 3PCF of galaxies depends on the shape of the linear power spectrum (Fry 1984; Jing \& Börner 1997) and the galaxy biasing relative to the underlying mass (Davis et al 1985; Gaztañaga \& Freiman 1994; Mo, Jing, \& White 1997; Matarrese, Verde, \& Heavens 1997; Catelan et al. 1998). It might also be sensitive to a possible non-Gaussianity of the initial density fluctuation (Fry \& Scherren 1994). Furthermore the 3PCF must be determined accurately if one wants to use the cosmic virial theorem to obtain the mean density of the Universe.

We have carried out a detailed analysis of the Las Campanas Redshift Survey (Shectman et al. 1996), and in this paper we report the measurement of the 3PCF of galaxies in this survey. We have not only measured the $3 \mathrm{PCF}$ in redshift space but also developed a method to measure the projected $3 \mathrm{PCF}$ which has simple relations to the real space 3PCF (§3.1). Our methods are checked very carefully with the help of mock catalogs generated from N-body simulations, and the physical meaning of these two quantities is also investigated (§3.2). Our statistical results are compared with previous work, with the emphasis on a critical examination of the hypothesis of the hierarchical form ( 33.4$)$. As we will see, the hierarchical form does not seem to be a good prescription even in the strong clustering regime. We will present a new fitting formula in $§ 3.3$. Implications for cosmogonic models are discussed in $\S 4$. 


\section{Observational sample and mock catalogs}

The sample used for our analysis is the Las Campanas Redshift Survey (Shectman et al. 1996; hereafter LCRS). This is the largest redshift survey, which is now publicly available. Our sample consists of all galaxies with recession velocities between 10,000 and $45,000 \mathrm{~km} \mathrm{~s}^{-1}$ and with absolute magnitudes (in the LCRS hybrid R band) between -18.0 and -23.0. There are 19558 galaxies in this sample, of which 9480 are in the three north slices and the rest in the three south slices. The survey is a well-calibrated sample of galaxies, ideally suited for statistical studies of large-scale structure. All known systematic effects in the survey are well quantified and documented (Shectman et al. 1996; Lin et al. 1996), and so most can be corrected easily in statistical analyses. The only exception is the 'fiber collision' limitation which prevents two galaxies in one $\sim 1.5 \times 1.5 \mathrm{deg}^{2}$ field from being observed when they are closer than $55^{\prime \prime}$ on the sky, because it is impossible to put fibers on both objects simultaneously. Here we will use extensively mock catalogs generated from N-body simulations to quantify this effect.

The real-space $2 \mathrm{PCF}$ and the Pairwise Velocity Dispersion (PVD) have been determined for the LCRS by JMB98. The redshift-space 2PCF and power spectrum for this sample were presented by Tucker et al. (1997) and Lin et al. (1997) respectively. All these studies have shown that the LCRS is large enough to accurately measure these low order statistical quantities. In particular, JMB98 have carried out a detailed comparison between the observed 2PCF and PVD and the predictions of currently favoured CDM cosmogonies. They have used a large set of mock samples to adequately compare models and observations. The construction of mock catalogues from the simulations, i.e. photometric catalogues subject to the same selection effects as the real observations are a very important aspect of their analysis, because only in this way could the statistical significance of the results be asserted. Three spatially flat models have been considered in JMB98, with $\left(\Omega_{0}, \lambda_{0}\right.$, $\left.\Gamma, \sigma_{8}\right)=(0.2,0.8,0.2,1),.(0.3,0.7,0.2,1$.$) , and (1.0,0.0,0.5,0.62)$, where $\Omega_{0}$ is the density parameter, $\lambda_{0}$ is the cosmological constant, $\Gamma=\Omega_{0} h$ and $\sigma_{8}$ are the shape parameter and normalization of the CDM power spectrum (Bardeen et al. 1986). All of the models give a steeper 2PCF, and a higher PVD on small scales than the data. Thus unless galaxies are biased with respect to the mass with a scale-dependent bias, all these models can be ruled out. Unfortunately physical models for a density or a (not so wanted, but perhaps unavoidable) velocity bias are not on firm grounds. Therefore in JMB98 a simple, but plausible phenomenological model for the bias has been suggested. To suppress the number of pairs in the DM distribution at small separations, it is assumed that the number of galaxies per unit dark matter mass $N / M$ is smaller in massive halos than in less massive ones. If a behaviour such as $N / M \propto M_{c l}^{\alpha}$ with $\alpha=-0.08$ is used for clusters of mass $M_{c l}$, the predictions of some CDM models are consistent with the observational results. The 
best agreement was achieved for the flat $\Omega_{0}=0.2$ model.

We will use 10 mock catalogs of this model to test our statistical methods and quantify the 'fiber collision' effect. Since this model has reproduced the LCRS 2PCF and PVD, we believe these mock catalogs are very suitable for this purpose. We shall also use these mock samples for model testing, as an example to illustrate the power of the three-point correlation function in discriminating between models which have similar two-point correlations. Since the model is a typical CDM model, we will generalize the discussion to other CDM models.

\section{The three point correlation function}

\subsection{Definitions and statistical methods}

The three-point correlation function $(3 \mathrm{PCF}) \zeta\left(r_{12}, r_{23}, r_{31}\right)$ is defined, through the joint probability $d P_{123}$ of finding one object simultaneously in each of the three volume elements $d \mathbf{r}_{1}, d \mathbf{r}_{2}$ and $d \mathbf{r}_{3}$ at positions $\mathbf{r}_{1}, \mathbf{r}_{2}$ and $\mathbf{r}_{3}$ respectively, as follows (P80):

$$
d P_{123}=\bar{n}\left(\mathbf{r}_{1}\right) \bar{n}\left(\mathbf{r}_{2}\right) \bar{n}\left(\mathbf{r}_{3}\right)\left[1+\xi\left(r_{12}\right)+\xi\left(r_{23}\right)+\xi\left(r_{31}\right)+\zeta\left(r_{12}, r_{23}, r_{31}\right)\right] d \mathbf{r}_{1} d \mathbf{r}_{2} d \mathbf{r}_{3}
$$

where $r_{i j}=\left|\mathbf{r}_{i}-\mathbf{r}_{j}\right|$, and $\bar{n}\left(\mathbf{r}_{i}\right)$ is the mean density of galaxies at $\mathbf{r}_{i}$. This definition can be applied straightforwardly to redshift surveys of galaxies to measure the $3 \mathrm{PCF} \zeta\left(s_{12}, s_{23}, s_{31}\right)$ of galaxies in redshift space (at this point we neglect the anisotropy induced by the redshift distortion which will be considered later). Here and below we use $\mathbf{r}$ to denote the real space and $\mathbf{s}$ the redshift space.

The 3PCF of galaxies can be measured from the counts of different triplets (P80). For this purpose, a sample of randomly distributed points, which has exactly the same boundaries and the same observational selection effects as the real survey, is generated. Four types of distinct triplets with triangles in the range $\left(s_{12} \pm 1 / 2 \Delta s_{12}, s_{23} \pm 1 / 2 \Delta s_{23}\right.$, and $\left.s_{31} \pm 1 / 2 \Delta s_{31}\right)$ are counted: the count $\operatorname{DDD}\left(s_{12}, s_{23}, s_{31}\right)$ of triplets formed by three galaxies; the count $\operatorname{DDR}\left(s_{12}, s_{23}, s_{31}\right)$ of triplets formed by two galaxies and one random point; the count $\operatorname{DRR}\left(s_{12}, s_{23}, s_{31}\right)$ of triplets formed by one galaxy and two random points; the count $R R R\left(s_{12}, s_{23}, s_{31}\right)$ of triplets formed by three random points. Following the definition $[\mathrm{eq}(2)]$, we shall use the following estimator

$$
\begin{aligned}
\zeta\left(s_{12}, s_{23}, s_{31}\right)= & \frac{27 R R R^{2}\left(s_{12}, s_{23}, s_{31}\right) \times D D D\left(s_{12}, s_{23}, s_{31}\right)}{D R R^{3}\left(s_{12}, s_{23}, s_{31}\right)} \\
& -\frac{9 R R R\left(s_{12}, s_{23}, s_{31}\right) \times D D R\left(s_{12}, s_{23}, s_{31}\right)}{D R R^{2}\left(s_{12}, s_{23}, s_{31}\right)}+2
\end{aligned}
$$


to measure the 3PCF of the galaxies in redshift space. The above formula is slightly different from the estimator used by Groth \& Peebles (1977). Here we have extended the argument of Hamilton (1993) for the 2PCF to the case of the 3PCF. The coefficients 27 and 9 are due to the fact that only distinct triplets are counted in this paper. Since the early work of Peebles and coworkers (P80) indicates that the $3 \mathrm{PCF}$ of galaxies is approximately hierarchical, it is convenient to express the $3 \mathrm{PCF}$ in a normalized form $Q_{\text {red }}\left(s_{12}, s_{23}, s_{31}\right)$ :

$$
Q_{\text {red }}\left(s_{12}, s_{23}, s_{31}\right)=\frac{\zeta\left(s_{12}, s_{23}, s_{31}\right)}{\xi\left(s_{12}\right) \xi\left(s_{23}\right)+\xi\left(s_{23}\right) \xi\left(s_{31}\right)+\xi\left(s_{31}\right) \xi\left(s_{12}\right)} .
$$

It is also convenient to use the variables introduced by Peebles (P80) to describe the shape of the triangles formed by the galaxy triplets. For a triangle with the three sides $s_{12} \leq s_{23} \leq s_{31}, s, u$, and $v$ are defined as:

$$
s=s_{12}, \quad u=\frac{s_{23}}{s_{12}}, \quad v=\frac{s_{31}-s_{23}}{s_{12}} .
$$

Clearly, $u$ and $v$ characterize the shape and $s$ the size of a triangle. We take equal logarithmic bins for $s$ and $u$ with the bin intervals $\Delta \lg s=\Delta \lg u=0.2$, and equal linear bins for $v$ with $\Delta v=0.2$. For our analysis, we take the following ranges for $s, u$ and $v$ : $0.63 \leq s \leq 10 h^{-1} \mathrm{Mpc}$ ( 6 bins); $1 \leq u \leq 4$ (3 bins); and $0 \leq v \leq 1$ (5 bins).

A sample of 25,000 random points is first generated. The counts $R R R$ are less than $\sim 5$ for small triangles $\left(s<1 h^{-1} \mathrm{Mpc}\right)$. In order to suppress the fluctuation induced by the random samples, we have recalculated the counts $R R R$ for $s_{31} \leq 4 h^{-1} \mathrm{Mpc}$ by generating a random sample 10 times larger, which ensure that the counts $R R R$ are at least $\sim 300$ of the interested triangle configurations. We scaled these counts to 25,000 random points and also use these counts to get $D R R$ on the small scales since $R R R / D R R$ is constant. However, it is not trivial to search triplets for so many points. We have generalized the ordinary linked-list technique of $\mathrm{P}^{3} \mathrm{M}$ simulations (Hockney \& Eastwood 1980) to spherical coordinates to count the triplets. The linked-list cells are specified by the spherical coordinates, i.e. the right accession $\alpha$, the declination $\delta$ and the distance $s$. With this short-range searching technique, we can avoid the triplets out of the range specified thus making counting triplets very efficient.

The $3 \mathrm{PCF}$ in redshift space $Q_{\text {red }}(s, u, v)$ depends both on the real space distribution of galaxies and on their peculiar motions. Although this information contained in $Q_{r e d}(s, u, v)$ is also useful for the study of the large scale structures (see $\S 4$ ), it is apparent that $Q_{\text {red }}(s, u, v)$ is different from $Q(r, u, v)$ in real space. In analogy with the analysis for the two-point correlation function, we have determined the projected three-point correlation function $\Pi\left(r_{p 12}, r_{p 23}, r_{p 31}\right)$. We define the redshift space three-point correlation function 
$\zeta_{z}\left(r_{p 12}, r_{p 23}, r_{p 31}, \pi_{12}, \pi_{13}\right)$ through:

$$
\begin{aligned}
d P_{123}^{z}= & \bar{n}\left(\mathbf{s}_{1}\right) \bar{n}\left(\mathbf{s}_{2}\right) \bar{n}\left(\mathbf{s}_{3}\right)\left[1+\xi_{z}\left(r_{p 12}, \pi_{12}\right)+\xi_{z}\left(r_{p 23}, \pi_{23}\right)+\xi_{z}\left(r_{p 31}, \pi_{31}\right)\right. \\
& \left.+\zeta_{z}\left(r_{p 12}, r_{p 23}, r_{p 31}, \pi_{12}, \pi_{13}\right)\right] d \mathbf{s}_{1} d \mathbf{s}_{2} d \mathbf{s}_{3}
\end{aligned}
$$

where $d P_{123}^{z}$ is the joint probability of finding one object simultaneously in each of the three volume elements $d \mathbf{s}_{1}, d \mathbf{s}_{2}$ and $d \mathbf{s}_{3}$ at positions $\mathbf{s}_{1}, \mathbf{s}_{2}$ and $\mathbf{s}_{3} ; \xi_{z}\left(r_{p}, \pi\right)$ is the redshift space two-point correlation function; $r_{p i j}$ and $\pi_{i j}$ are the separations of objects $i$ and $j$ perpendicular to and along the line-of-sight respectively. The projected 3PCF $\Pi\left(r_{p 12}, r_{p 23}, r_{p 31}\right)$ is then defined as:

$$
\Pi\left(r_{p 12}, r_{p 23}, r_{p 31}\right)=\int \zeta_{z}\left(r_{p 12}, r_{p 23}, r_{p 31}, \pi_{12}, \pi_{13}\right) d \pi_{12} d \pi_{23}
$$

Because the total amount of triplets along the line-of-sight is not distorted by the peculiar motions, the projected $3 \mathrm{PCF} \Pi\left(r_{p 12}, r_{p 23}, r_{p 31}\right)$ is related to the $3 \mathrm{PCF}$ in real space $\zeta\left(r_{12}, r_{23}, r_{31}\right)$ :

$$
\Pi\left(r_{p 12}, r_{p 23}, r_{p 31}\right)=\int \zeta\left(\sqrt{r_{p 12}^{2}+y_{12}^{2}}, \sqrt{r_{p 23}^{2}+y_{23}^{2}}, \sqrt{r_{p 31}^{2}+\left(y_{12}+y_{23}\right)^{2}}\right) d y_{12} d y_{23} .
$$

Similarly as for $\zeta\left(s_{12}, s_{23}, s_{31}\right)$, we measure $\zeta_{z}\left(r_{p 12}, r_{p 23}, r_{p 31}, \pi_{12}, \pi_{13}\right)$ by counting the numbers of triplets $D D D\left(r_{p 12}, r_{p 23}, r_{p 31}, \pi_{12}, \pi_{13}\right), D R R\left(r_{p 12}, r_{p 23}, r_{p 31}, \pi_{12}, \pi_{13}\right)$, $R D D\left(r_{p 12}, r_{p 23}, r_{p 31}, \pi_{12}, \pi_{13}\right)$ and $R R R\left(r_{p 12}, r_{p 23}, r_{p 31}, \pi_{12}, \pi_{13}\right)$ formed by galaxies and/or random points with the projected separations $r_{p 12}, r_{p 23}$, and $r_{p 31}$ and radial separations $\pi_{12}$ and $\pi_{23}$. We will use $r_{p}, u$ and $v$ :

$$
r_{p}=r_{p 12}, \quad u=\frac{r_{p 23}}{r_{p 12}}, \quad v=\frac{r_{p 31}-r_{p 23}}{r_{p 12}} .
$$

to quantify a triangle with $r_{p 12} \leq r_{p 23} \leq r_{p 31}$ on the projected plane. Equal logarithmic bins of intervals $\Delta \lg r_{p}=\Delta \lg u=0.2$ are taken for $r_{p}$ and $u$, and equal linear bins of $\Delta v=0.2$ for $v$. The same ranges of $u$ and $v$ are used as for $\zeta(s, u, v)$, but $r_{p}$ is from $0.128 h^{-1} \mathrm{Mpc}$ to $4 h^{-1} \mathrm{Mpc}\left(7\right.$ bins). The radial separations $\pi_{12}$ and $\pi_{23}$ are from $-25 h^{-1} \mathrm{Mpc}$ to $25 h^{-1} \mathrm{Mpc}$ with a bin size of $1 h^{-1} \mathrm{Mpc}$. The projected 3PCF is estimated by summing up $\zeta_{z}\left(r_{p}, u, v, \pi_{12}^{i}, \pi_{23}^{j}\right)$ at different radial bins $\left(\pi_{12}^{i}, \pi_{23}^{j}\right)$ :

$$
\Pi\left(r_{p}, u, v\right)=\sum_{i, j} \zeta_{z}\left(r_{p}, u, v, \pi_{12}^{i}, \pi_{23}^{j}\right) \Delta \pi_{12}^{i} \Delta \pi_{23}^{j}
$$

and normalized as

$$
Q_{p r o j}\left(r_{p}, u, v\right)=\frac{\Pi\left(r_{p}, u, v\right)}{w\left(r_{p 12}\right) w\left(r_{p 23}\right)+w\left(r_{p 23}\right) w\left(r_{p 31}\right)+w\left(r_{p 31}\right) w\left(r_{p 12}\right)} .
$$


where $w\left(r_{p}\right)$ is the projected two-point correlation function (Davis \& Peebles 1983; JMB98)

$$
w\left(r_{p}\right)=\sum_{i} \xi_{z}\left(r_{p}, \pi^{i}\right) \Delta \pi^{i}
$$

An interesting property of the projected $3 \mathrm{PCF}$ is that if the three-point correlation function is of the hierarchical form, the normalized function $Q_{p r o j}\left(r_{p}, u, v\right)$ is not only a constant but also equal to $Q$. Therefore the measurement of $Q_{\text {proj }}\left(r_{p}, u, v\right)$ can be used to test the hierarchical form which was proposed mainly based on the analysis of angular catalogs.

\subsection{N-body tests of the statistical methods}

To test the reliability of our statistical analysis and to demonstrate the effects of the redshift distortion, the projection, and the fiber collisions, we make use of the full simulation and the mock catalogs. Because the mock samples are cluster-(under)weighted, we have applied the same weighting to the full simulation to achieve a proper comparison. To calculate the quantities in redshift space for the full simulation, we assume that the third axis is along the line-of-sight.

In Fig. (1) we compare $Q_{r e d}(s, u, v)$ estimated with our statistical method from the mock samples with the true value. The latter is determined from the full simulation using the method of Jing \& Börner (1997). On the scales from $1 h^{-1} \mathrm{Mpc}$ to $10 h^{-1} \mathrm{Mpc}$, the two estimated quantities agree fairly well, indicating that the LCRS can yield an unbiased estimate within the error bars of the $3 \mathrm{PCF}$ in redshift space. The test is important, considering the fact that the LCRS is essentially two-dimensional with one dimension in the direction of the line-of-sight.

Fig. (2) shows the projected $Q_{\text {proj }}\left(r_{p}, u, v\right)$ estimated from the mock samples with the method described above. The true $Q_{\text {proj }}\left(r_{p}, u, v\right)$ can be calculated from the real space $3 \mathrm{PCF}$ $\zeta(r, u, v)$ through Eq. (8). We determine $\zeta(r, u, v)$ for the full simulation using the method of Jing \& Börner (1997) and calculate the integral of Eq.(8) by linearly interpolating the estimated $\zeta(r, u, v)$. The two estimated quantities agree very well within the error bars, indicating that our method can give a correct estimate of the projected 3PCF. The real space $3 \mathrm{PCF} Q(r, u, v)$ is also shown in the figure by the thick lines. It decreases with the scale $r$, as noted previously (Matsubara \& Sutd 1994; Jing \& Börner 1997). The reason for the decrease is due to the fact that the slope of the power spectrum is more negative on smaller scales (see Jing, in preparation, for a detailed discussion). The consequence is that due to the averaging of $Q(r, u, v)$ on scales $r \geq r_{p}$ [Eq.(8)], the projected $Q_{\text {proj }}\left(r_{p}, u, v\right)$ is also a decreasing function of $r_{p}$ but smaller than $Q(r, u, v)$ for $r=r_{p}$ (compare thick and thin lines in the figure). Another interesting point is that $Q(r, u, v)$ is much higher than its 
counterpart in redshift space $Q_{r e d}(s, u, v)$ on scales $\lesssim 10 h^{-1} \mathrm{Mpc}$. This result is well-known since the redshift distortion smears out the dense clusters and thus reduces the $3 \mathrm{PCF}$ on small scales (e.g. Matsubara \& Suto 1994; Matsubara 1994).

We have also tested for the fiber collision effect of the LCRS sample as we did for the $2 \mathrm{PCF}$ and PVD in JMB98. While both the 2PCF $\left[\xi(s)\right.$ and $\left.w\left(r_{p}\right)\right]$ and the 3PCF $[\zeta(s, u, v)$ and $\left.\Pi\left(r_{p}, u, v\right)\right]$ show some small dependence on this effect, it cancels out completely, when we divide these two quantities by one another to form the normalized $3 \mathrm{PCF} Q_{\text {red }}(s, u, v)$ and $Q_{\text {proj }}\left(r_{p}, u, v\right)$. Therefore, the fiber collisions of the LCRS have little effect on the normalized functions $Q_{r e d}(s, u, v)$ and $Q_{p r o j}\left(r_{p}, u, v\right)$.

In summary, these tests have convinced us that our method is suitable for giving a stable measurement of the $3 \mathrm{PCF}$ from the LCRS with reliable error estimates.

\subsection{The statistical results of the LCRS}

We present our results of the $3 \mathrm{PCF}$ in redshift space $Q_{r e d}(s, u, v)$ and of the projected 3PCF $Q_{p r o j}\left(r_{p}, u, v\right)$ in Figures (3) and (4) respectively for the Las Campanas Redshift Survey. The errors of the $Q$-values are the bootstrap errors which are estimated with the approximate formula of Mo, Jing, \& Börner (1992). As we can see from Fig. (3), the 3PCF obtained from redshift space is not changing very much with $s$ or $u$; it increases somewhat with $v$. For small $v, Q_{\text {red }}$ is approximately constant with a value of $\sim 0.5$, but it increases up to $\sim 1$ when $v \approx 1$. Compared with the $3 \mathrm{PCF}$ in redshift space, the projected one $Q_{p r o j}\left(r_{p}, u, v\right)$ [Figure (国)] shows quite similar dependences on triangle shape (i.e. $u$ and $v$ ), but quite a different dependence on the triangle size (i.e. $s$ or $r_{p}$ ), though the errors of the projected $3 \mathrm{PCF}$ are larger. Its value decreases with $r_{p}$ from about 1.2 at $r_{p}=0.2 h^{-1} \mathrm{Mpc}$ to 0.5 at $r_{p}$ about $2 h^{-1} \mathrm{Mpc}$. Both this decrease with growing $r_{p}$ and the weak increase with $v$ are in contrast to the hierarchical assumption. If the three-point correlation function in real space were hierarchical, equation (8) shows that the projected one would also be hierarchical and equal to $Q$. This behavior, however, is qualitatively in agreement with the CDM model predictions, and we will discuss this point in $\S 4$.

The hierarchical form (eq.1) does not seem to provide an adequate description of our results of the $3 \mathrm{PCF}$ in redshift space $Q_{r e d}(s, u, v)$ or of the projected $3 \mathrm{PCF} Q_{p r o j}\left(r_{p}, u, v\right)$. We have looked for fitting formulae for both quantities. Our results can be fitted quite well by $Q_{r e d}(s, u, v)=0.5 \cdot 10^{\left[0.2+0.1\left(\frac{s}{s+1}\right)^{2}\right] v^{2}}$ and $Q_{p r o j}\left(r_{p}, u, v\right)=0.7 r_{p}^{-0.3}\left(s\right.$ and $r_{p}$ are in unit of $h^{-1} \mathrm{Mpc}$ ), which are the smooth lines on Figure (河) and the thick lines on Figure (4). We have neglected the weak systematic dependence on $v$ of $Q_{p r o j}\left(r_{p}, u, v\right)$ at $r_{p} \gtrsim 1 h^{-1} \mathrm{Mpc}$ 
in fitting this quantity since the dependence is not statistically significant on the scales we probed. We do not intend to give an error estimate for the coefficients in the formulae, since the errors of the $Q$-values in individual bins are likely non-Gaussian distributed and correlated among different bins. These formulae are intended to give a simple, but for most purposes accurate, approximation to our statistical results.

Since the real space $3 \mathrm{PCF} \zeta(r, u, v)$ possibly depends on $r, u$ and $v$ in a complicated way, the inversion of eq.(8) to get $\zeta(r, u, v)$ from the projected $\Pi\left(r_{p}, u, v\right)$ is certainly unstable. We have noted however that the projected $Q_{\text {proj }}\left(r_{p}, u, v\right)$ can be modeled very well by half the value of the mock projected $Q_{p r o j}^{c d m}\left(r_{p}, u, v\right)$ [the thin lines in Fig. (四)], which means that $Q(r, u, v)=0.5 Q^{c d m}(r, u, v)$, where $Q^{c d m}(r, u, v)$ is the real-space 3PCF of the CDM model, is a good approximation to the real-space $3 \mathrm{PCF}$ of LCRS galaxies on scales $\lesssim 3 h^{-1} \mathrm{Mpc}$. These $Q(r, u, v)$ can be easily read out from the $Q^{c d m}(r, u, v)$ in Fig. (2).

\subsection{Discussion}

The high quality of the LCRS survey, by its CCD photometry, its complete redshift information and its large size, has enabled us to give a reliable determination of the 3PCF. It is the first time that the three-point correlation functions both in redshift space and in real (projected) space can be measured as a function of the triangle size and shape, with only a fractional error in each individual bin, for a wide range of triangle configurations. Although our statistical results in real space on scales $\lesssim 1 h^{-1} \mathrm{Mpc}$ are not far from the hierarchical prediction (1) with $Q=1.3 \pm 0.2$ (Groth \& Peebles 1977; GP77), the systematic changes of $Q_{\text {proj }}\left(r_{p}, u, v\right)$ with the triangle configurations clearly point to a more elaborate model for $Q(r, u, v)$. One such model was already proposed in $§ 3.3$. Although our result of $Q_{\text {red }}(s, u, v)$ generally agrees with the previous studies based on much smaller redshift samples (see §1), our measurement has much better accuracy.

Recently there are concerns about the reliability of the high-order correlation functions derived from photographic-plates based galaxy catalogs. The skewness of the galaxies derived from the Automatic Plate Machine (APM) angular catalog (Maddox et al. 1990) and from the Edinburgh/Durham Southern Galaxy Catalogue (EDSGC, Heydon-Dumbleton, Collins, \& MacGillivray 1989) is significantly different even on small scales $<1 h^{-1} \mathrm{Mpc}$ (Gaztañaga 1994; Szapudi, Meiksin, \& Nicho 1996), despite the fact that both catalogs are constructed from the same UK IIIa-J Schmidt photographic plates and the latter is just a sub-sample $(\sim 1 / 4)$ of the former. The difference seems to arise from their different methods to digitalize the plates (Szapudi \& Gaztañaga 1998), and at this point it is rather difficult to judge which method is superior. It is also worth noting that the $3 \mathrm{PCF}$ results 
of GP77 are somewhat sensitive to the corrections they have applied to the Lick catalog. Fortunately the LCRS survey does not suffer from these uncertainties since its photometric catalog was constructed from the CCD drift scans. Furthermore in our analysis we did not have to apply any additional corrections except for those well-documented by the survey team.

It is interesting to compare our results with the skewness $S_{3}(R)$ determined from the large angular catalogs, in particular since the two studies based on the APM and EDSCG catalogs have yielded rather discrepant results. The skewness is related to the $3 \mathrm{PCF}$ through an integral as follows:

$$
\begin{aligned}
S_{3}(R) & =\frac{\bar{\zeta}(R)}{\bar{\xi}^{2}(R)} \\
\bar{\zeta}(R) & =\frac{1}{V^{3}} \int_{\text {sphere } R} d \mathbf{r}_{\mathbf{1}} d \mathbf{r}_{\mathbf{2}} d \mathbf{r}_{\mathbf{3}} \zeta\left(r_{12}, r_{23}, r_{31}\right) \\
\bar{\xi}(R) & =\frac{1}{V^{2}} \int_{\text {sphere } R} d \mathbf{r}_{\mathbf{1}} d \mathbf{r}_{\mathbf{2}} \xi\left(r_{12}\right)
\end{aligned}
$$

where $V=\frac{4 \pi}{3} R^{3}$. It needs full information of $Q(r, u, v)$ to calculate the skewness [eq. (13)], and we use the model proposed in $\S 3.3$. The skewness $S_{3}(R)$ for the LCRS survey is then about 4.5 at $R=0.2 h^{-1} \mathrm{Mpc}$ and about 3.5 at $R=1 h^{-1} \mathrm{Mpc}$, which seem in agreement with the results of Szapudi, Meiksin, \& Nichol (1996) based on the EDSGC catalog but significantly higher than the APM results of Gaztañaga (1994) on the scales $<1 h^{-1} \mathrm{Mpc}$.

The cosmic virial theorem (CVT) has been widely used to measure the mean density of the universe. If the $3 \mathrm{PCF}$ is hierarchical, the CVT can be expressed in its simplified form relating the density parameter $\Omega_{0}, \xi(r), Q$, and the PVD $\sigma_{12}(r)$. Since our results show that the $3 \mathrm{PCF}$ is not hierarchical, this relation becomes much more complicated and it is necessary to work out the integration over $\zeta\left(r_{12}, r_{23}, r_{31}\right)$ which might depend on $\zeta\left(r_{12}, r_{23}, r_{31}\right)$ on very small scales $r \sim 0$. Since $\zeta\left(r_{12}, r_{23}, r_{31}\right)$ is a decreasing function of the triangle size, previous studies which usually used the $Q$ value at $\sim 1 h^{-1} \mathrm{Mpc}$ might have overestimated the mean density. The size of galaxies, which are usually treated as point sources in the CVT application, may also be important in the estimate of the mean density, especially near $r \sim 0$ (Peebles 1976; Suto \& Jing 1997).

We would like to remark here that the significant difference between $Q_{\text {red }}$ and $Q_{\text {proj }}$ at small scales comes from peculiar motions of galaxies. This gives another possibility to estimate the velocity dispersion of galaxies (Matsubara 1994). 


\section{A case for model testing}

In this section we compare the 3PCF of the LCRS with model predictions. Jing \& Börner (1997) and Jing (in preparation) have recently studied the $3 \mathrm{PCF} Q(r, u, v)$ for a set of CDM models based on second-order perturbation theory and N-boby simulations. We found that for fixed $u$ and $v, Q(r, u, v)$ is a decreasing function of the size $r$. In the strongly non-linear regime $(\xi(r) \gg 1, Q(r, u, v)$ shows a very weak dependence on $u$ and $v$. In the weakly non-linear and linear regimes, $Q(r, u, v)$ increases significantly with $v$ for fixed $r$ and $u$. All these features are found in the projected $Q_{p r o j}\left(r_{p}, u, v\right)$ of the LCRS galaxies. Therefore the statistical results found from the LCRS survey are all qualitatively consistent with the mass $3 \mathrm{PCF}$ based on N-body simulations of cosmological models.

As an example to quantitatively test models with the $3 \mathrm{PCF}$, we compare the 3PCFs of the LCRS galaxies with the results of the mock samples in Figs. (5) and (6). Only in this way could the redshift distortion and the projection effects be accounted for properly. From the figures we find that the qualitative features, i.e. the dependence on $v$ for fixed $s$ or $r_{p}$, and $u$, the decrease of $Q$ with increasing values of $s$ or $r_{p}$ are reproduced quite well in the mock samples. The values of the data set, however, are consistently lower than the mean model predictions by a factor $\sim 2$. Since the $Q$-values in each bin of the ten mock samples are not Gaussian distributed (skewed to high values), it is not meaningful to use the standard deviation to quantify the statistical significance. Instead we pick up the lowest of the ten mock $Q_{\text {red }}$ or $Q_{\text {proj }}$ values in each bin and compare it with the observed results. The thick lines in Figs. (5) and (6) correspond to these lowest values for $u=1.29$, which should be compared with the open triangles. These lines are still higher than (in most bins) or at least as high as (in a few bins) the observational values, which indicates that the observational values are lower than this model's predictions at a confidence level $\gtrsim 90 \%$. The underlying model for the mock sample is a CDM universe with $\Omega_{0}=0.2$ and $\lambda_{0}=0.8$ and with clusters underweighted (see $\S 2$ ). We have computed the $3 \mathrm{PCF}$ for this universe without cluster weighting, and found the cluster weighting, like the fiber collision effect, does not change much the value of $Q(r, u, v)$. Thus, even if this model fits the $2 \mathrm{PCF}$ and the PVD of the LCRS galaxies quite well, it seems not really adequate to describe the clustering of galaxies when the $3 \mathrm{PCF}$ is considered, unless the observed $3 \mathrm{PCF}$ is biased low by the cosmic variance [at $\lesssim 10 \%$ probability]. This might indicate that the gravitational interaction alone is not sufficient to describe the clustering of galaxies, and physical processes of gas and radiation hydrodynamics connected with galaxy formation must be taken into account. A positive bias, i.e. a biasing parameter $b>1$, can reduce the 3PCF. But, perhaps this conclusion goes too far - in fact, a slightly higher shape parameter $\Gamma$ will give a better fit, because $Q$ becomes smaller if $\Gamma$ is increased. It appears that a model with a new set of parameters is to be sought and the $3 \mathrm{PCF}$ determined here should provide 
to such new models a test in addition to the $2 \mathrm{PCF}$ and the PVD.

\section{Conclusion}

The result is clear, and the conclusions are straightforward: We have succeeded in measuring the 3PCF from the LCRS. This is the first time that the three-point correlation function of galaxies has been measured accurately from a redshift survey. Both the 3PCF in redshift space and the projected $3 \mathrm{PCF}$ have been measured as a function of the triangle size and shape with only a fractional uncertainty in each individual bin. Various tests have been carried out to assure that the measurement is stable and that the errors are estimated reliably. Our results indicate that the $3 \mathrm{PCF}$ soth in redshift space and in real space have small but significant deviations from the well-known hierarchical form. The 3PCF in the redshift space can be fitted by $Q_{r e d}(s, u, v)=0.5 \cdot 10^{\left[0.2+0.1\left(\frac{s}{s+1}\right)^{2}\right] v^{2}}$ for $0.8<s_{12}<8 h^{-1} \mathrm{Mpc}$ and $s_{31}<16 h^{-1} \mathrm{Mpc}$, and the projected $3 \mathrm{PCF}$ by $Q_{p r o j}\left(r_{p}, u, v\right)=0.7 r_{p}^{-0.3}$ for $0.2<r_{p 12}<3 h^{-1} \mathrm{Mpc}$ and $r_{p 31}<6 h^{-1} \mathrm{Mpc}\left(s\right.$ and $r_{p}$ are in unit of $\left.h^{-1} \mathrm{Mpc}\right)$. Although it might be not unique to get 3-D real space $Q(r, u, v)$ from the measured projected function $Q_{\text {proj }}\left(r_{p}, u, v\right)$, we found that a half of the predicted $Q(r, u, v)$ of the CDM model considered in this paper provides a good description of the LCRS data.

The three-point correlation function gives an additional statistical tool to constrain cosmogonic models. The general dependence of the 3PCF on triangle shape and size is in qualitative agreement with the CDM cosmogonic models. Quantitatively the 3PCF of the models may depend on the biasing parameter and the shape of the power spectrum, in addition to other model parameters. Taking our result together with the constraints imposed by the two-point correlation function and the pairwise velocity dispersion of galaxies also obtained from the LCRS, we find that we have difficulties to produce a simple model that meets all constraints perfectly. Among the CDM models considered, the flat model with $\Omega=0.2$ meets the $2 \mathrm{PCF}$ and PVD constraints, but gives higher values for the $3 \mathrm{PCF}$ than observed. This may indicate that more sophisticated bias models or a more sophisticated combination of model parameters must be considered.

We are grateful to Yasushi Suto for helpful discussions, and for the hospitality extended to us at the physics department of Tokyo university. G. B. thanks the Yamada foundation for support during his stay at RESCEU. J.Y.P. gratefully acknowledges the receipt of a JSPS postdoctoral fellowship. Support by SFB375 is also acknowledged. The simulations were carried out on VPP/16R and VX/4R at the Astronomical Data Analysis Center of the National Astronomical Observatory, Japan. 


\section{REFERENCES}

Bardeen J., Bond J.R., Kaiser N., Szalay A.S., 1986, ApJ, 304, 15

Baugh C.M., 1996, MNRAS, 280, 267

Bean A.J., Efstathiou G., Ellis R.S., Peterson B.A., Shanks T., 1983, MNRAS, 205, 605

Bouchet, F.R., Strauss, M. A., Davis, M., Fisher, K. B., Yahil, A., Huchra, J. P., 1993, ApJ, 417, 36

Catelan. P., Lucchin, F., Matarrese, S., Porciani, C. 1997, MNRAS (submitted); astro-ph/9708067

Davis M., Peebles P.J.E., 1983, ApJ, 267, 465

Davis, M., Efstathiou, G., Frenk, C. S., \& White, S. D. M. 1985, ApJ, 292, 371

Fry J.N., 1984, ApJ, 279, 499

Fry J.N., Scherrer R.J., 1994, ApJ, 429, 36

Gaztañaga E. 1994 ApJ, MNRAS, 268, 913

Gaztañaga E., Freiman J.A., 1994, ApJ, 437, L13

Hamilton A.J.S., 1993, ApJ, 417, 19

Hale-Sutton, D., Fong, R., Metcalfe, N., Shanks, T. 1989, MN, 237, 569

Hermit, S. et al., 1996, MN, 283, 709

Hockney R.W., Eastwood J.W., 1981, Computer simulations using particles. McGraw-Hill Inc., New York

Heydon-Dumbleton, N.H., Collins, C.A., \& MacGillivray, H.T. 1989, MNRAS, 238,379

Efstathiou, G., \& Jedrzejewski, R.I. 1984, Adv. Space Res., 3, 379

Groth, E.J., \& Peebles, P.J.E. 1977, ApJ, 217, 385

Jain B. 1997, MNRAS, 287, 687

Jing, Y.P., Mo, H.J., \& Börner, G. 1991, A\&A, 252, 449

Jing, Y.P., Börner, G. 1997, A\&A, 318, 667 
Jing Y.P., Mo, H.J., Börner G., 1998, ApJ, 494, ??? (astro-ph/9707106)

Lauberts, A. 1982, The ESO-Uppsala Survey of the ESO(B) Atlas, European Southern Observatory

Lin H. et al. 1996, ApJ, 464, 60

Lin H. et al. 1997, ApJ, 471, 617

Lin H. et al. 1998, in preparation

Matarrese, S., Verde, L., Heavens, A. 1997, MNRAS (in press); astro-ph/9706059

Maddox S.J. Efstathiou G., Sutherland W.J., Loveday, J. 1990, MNRAS, 243, 692

Matsubara T., Suto Y., 1994, ApJ, 420, 497

Matsubara T. 1994, ApJ, 424, 30

Mo, H.J., Jing, Y.P., \& Börner, G. 1992, ApJ, 392, 452

Mo, H.J., Jing, Y.P., White, S.D.M., 1997, MNRAS, 284, 189

Peebles, P.J.E. 1976, ApSS, 45, 3

Peebles P.J.E., 1980, The Large-Scale Structure of the Universe, Princeton University Press, Princeton

Peebles, P.J.E. 1981, in Annals New York Academy of Science, 157

Ratcliffe A., Shanks T., Parker Q.A., Fong R., 1997, preprint (astro-ph/9702227)

Shectman S. A., Landy, S.D., Oemler A., Tucker D.L., Lin H., Kirshner R.P., Schechter P.L., 1996, ApJ, 470, 172

Suto Y., Jing Y.P. 1997, ApJS, 110, 167

Strauss, M.A., Willick, J. A. 1995, Phys. Rep., 261, 271

Szapudi I., Meiksin A., Nichol R.C. 1996, ApJ473, 16

Szapudi I., Gaztañaga E. 1998, MNRAS (submitted); astro-ph/9712156

Tucker, D. et al. 1997, MNRAS, 285, L5

Yano, T., Gouda, N. 1997, ApJ, 487, 473 
This preprint was prepared with the AAS $\mathrm{LAT}_{\mathrm{E}} \mathrm{X}$ macros v4.0. 


\section{Comparison of mock to full simulation}

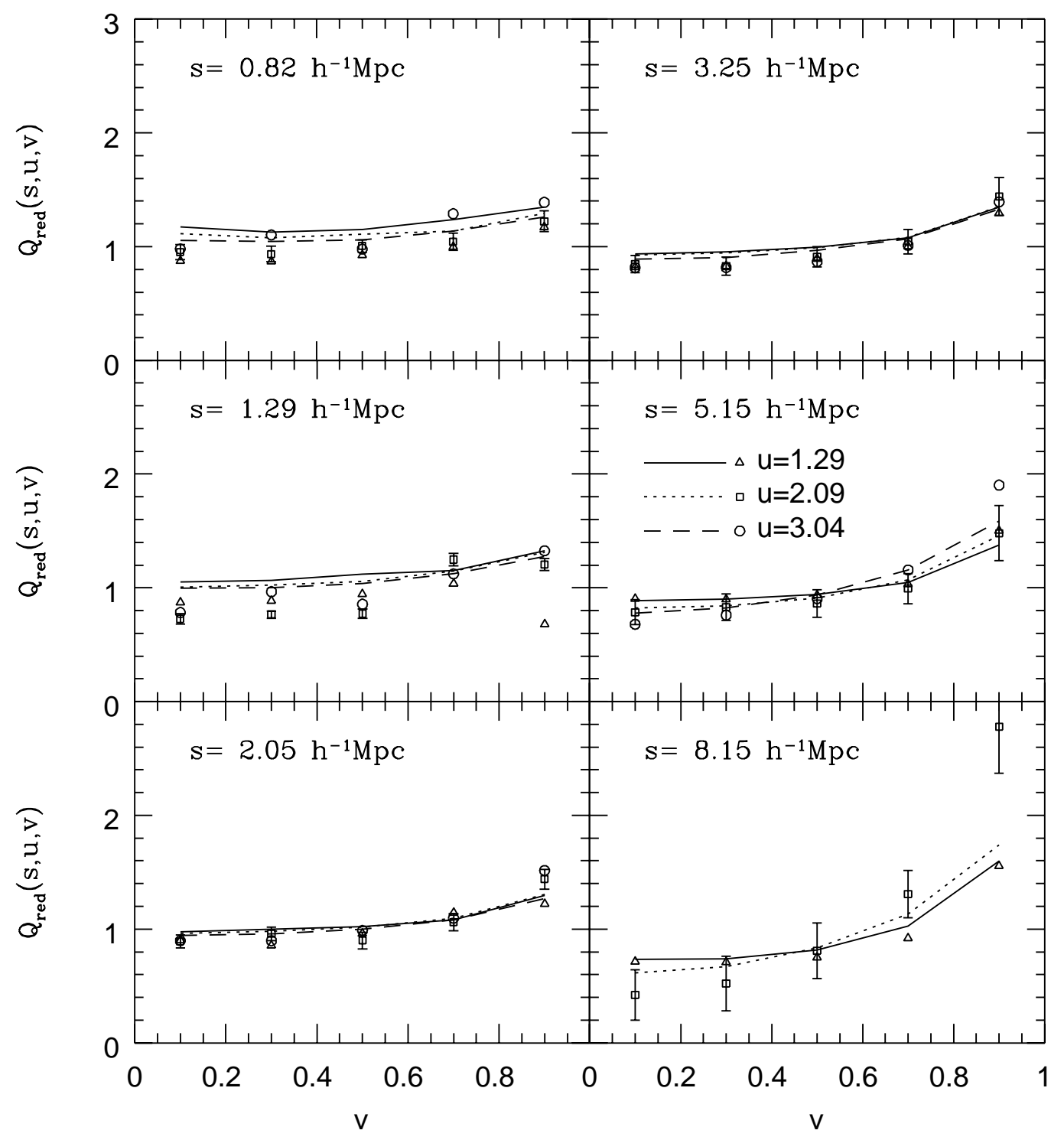

Fig. 1. - The normalized $3 \mathrm{PCF}$ in redshift space $Q_{\text {red }}(s, u, v)$ of the mock samples (symbols) and of the full simulation (lines). The error bars are the $1 \sigma$ standard deviation of the measurement for the 10 mock samples. For clarity, the error bars are plotted for $u=2$ only but those for the other two values of $u$ are very similar. 
Comparison of mock to full simulation

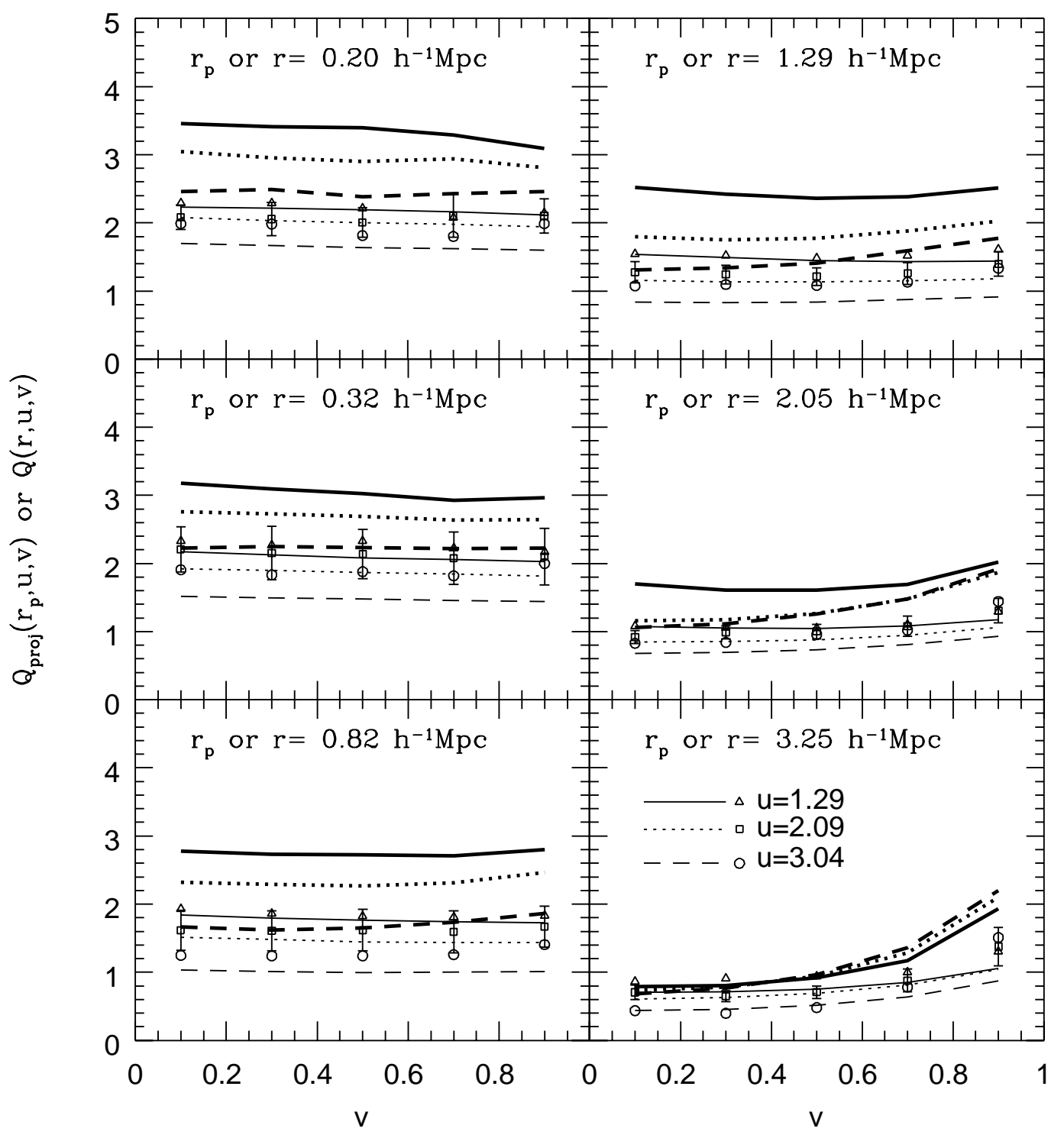

Fig. 2.- The normalized projected $Q_{\text {proj }}\left(r_{p}, u, v\right)$ of the mock samples (symbols) and of the full simulation (thin lines). The latter is computed through eq. (8) from the 3-D 3PCF $Q(r, u, v)$ of the full simulation which are also plotted with the thick lines. A comparison of the symbols and the thin lines tests the statistical methods, and a comparison of the thin lines and thick lines shows the projection effect. As in Fig. (1), the error bars are the $1 \sigma$ standard deviation of the measurement for the 10 mock samples and are plotted for $u=2$ only. 
Redshift-space 3PCF of the LCRS Survey

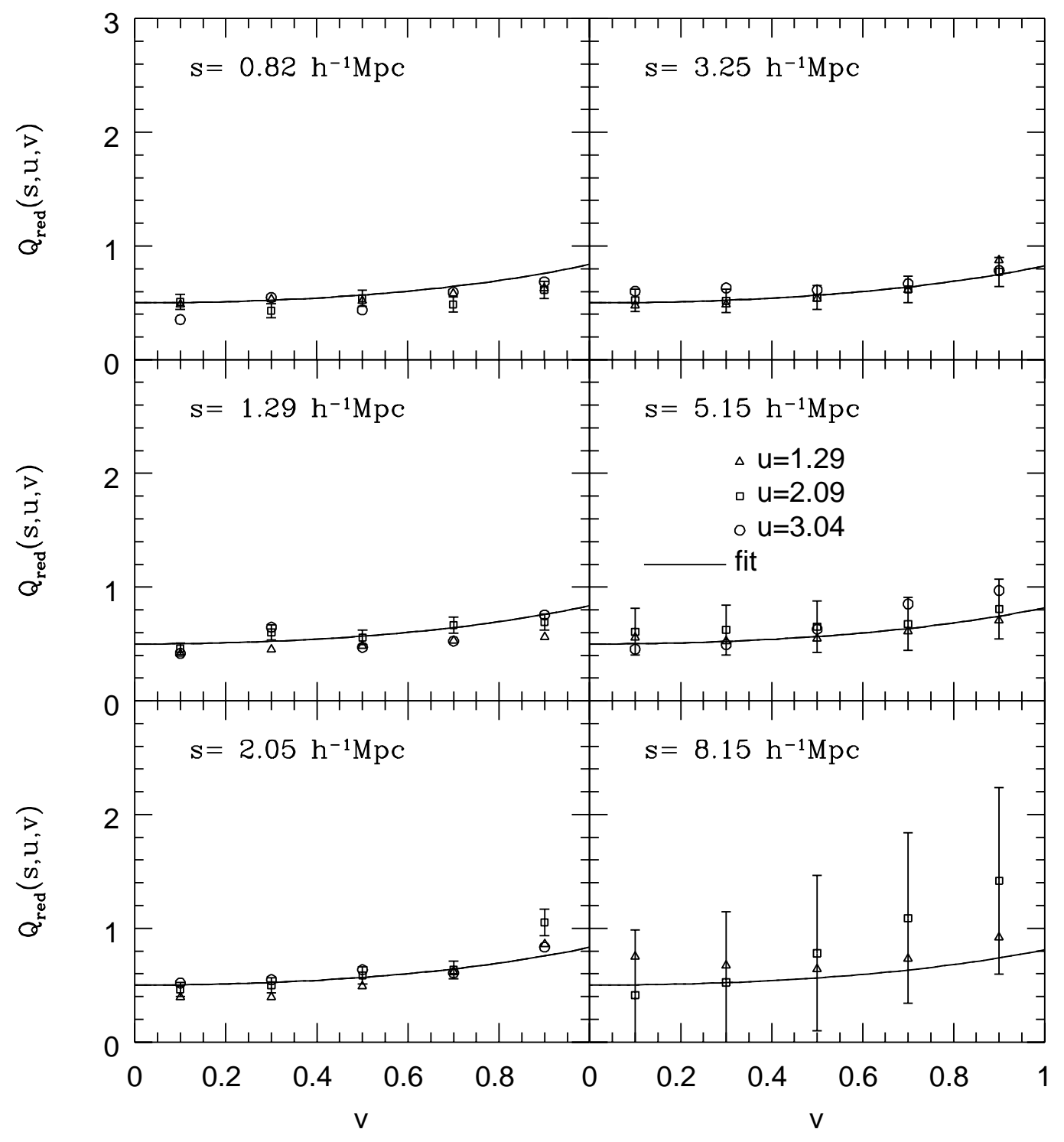

Fig. 3.- The normalized $3 \mathrm{PCF}$ in redshift space $Q_{\text {red }}(s, u, v)$ of the LCRS survey (symbols). The errors are estimated by the bootstrap resampling method. For clarity, the error bars are plotted for $u=2$ only but those for the other two values of $u$ are very similar. The results are well fit by $Q_{r e d}(s, u, v)=0.5 \cdot 10^{\left[0.2+0.1\left(\frac{s}{s+1}\right)^{2}\right] v^{2}}\left(s\right.$ is unit of $\left.h^{-1} \mathrm{Mpc}\right)$ which are shown by the solid lines. 


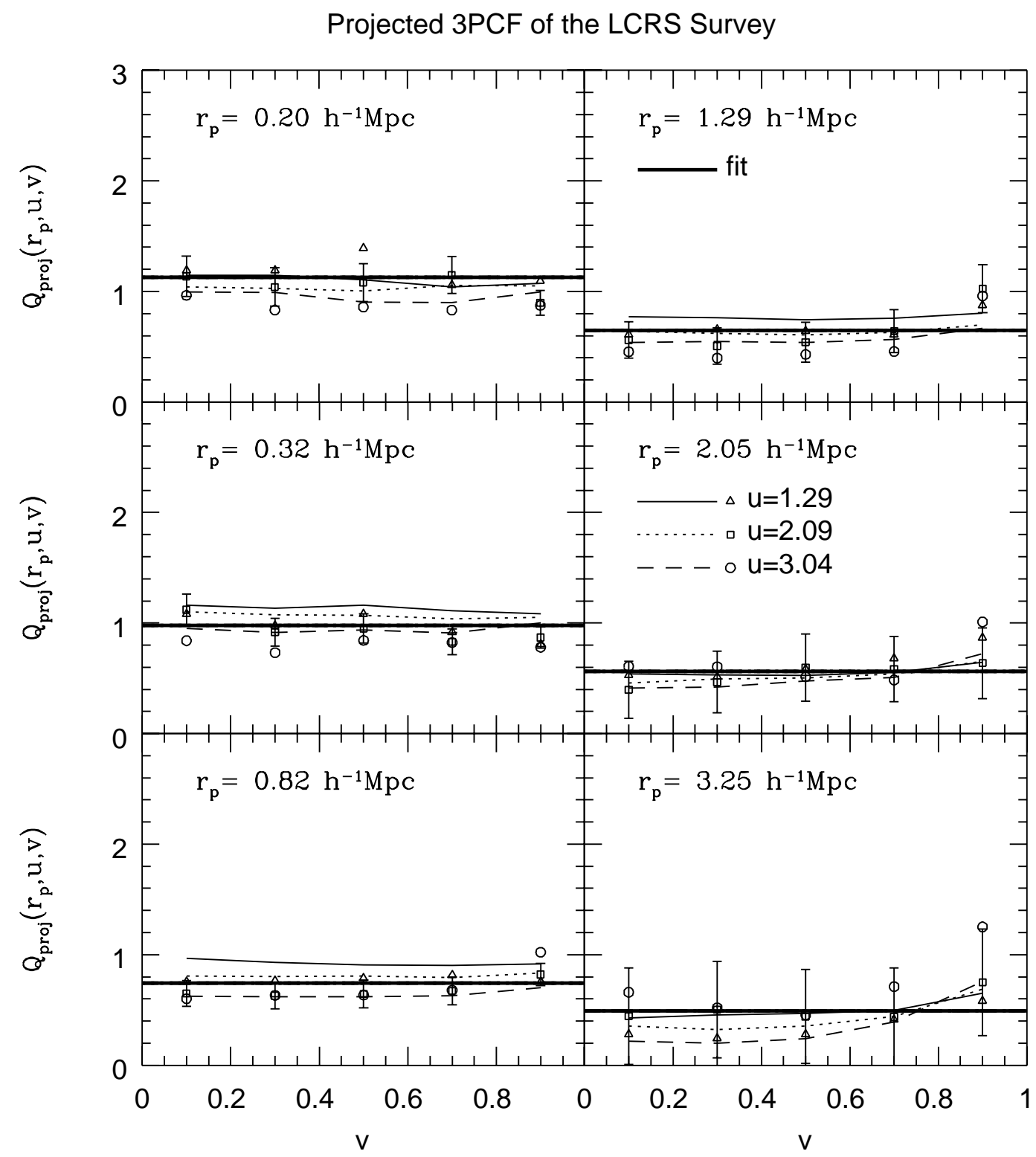

Fig. 4.- The normalized projected $3 \mathrm{PCF} Q_{p r o j}\left(r_{p}, u, v\right)$ of the LCRS survey (symbols). The errors are estimated by the bootstrap resampling method. For clarity, the error bars are plotted for $u=2$ only but those for the other two values of $u$ are very similar. The results are well fit by $Q_{p r o j}\left(r_{p}, u, v\right)=0.7\left(r_{p}\right)^{-0.3}\left(r_{p}\right.$ is unit of $\left.h^{-1} \mathrm{Mpc}\right)$ which are shown by the thick solid lines. The thin lines are a half of the mean projected $3 \mathrm{PCF}$ of the the mock samples, which seem to fit the observational data very well. 


\section{Comparison of the LCRS to the Model}



Fig. 5.- The normalized $3 \mathrm{PCF}$ in redshift space $Q_{\text {red }}(s, u, v)$ of the LCRS survey (open symbols) compared with the mean value of the ten mock samples (solid symbols). The thick solid lines represent the lowest of the ten mock $Q_{\text {red }}(s, u, v)$ values in each $u=1.29$ bin, which can be regarded as the lower model limits at $\sim 90 \%$ significance level. 
Comparison of the LCRS to the Model

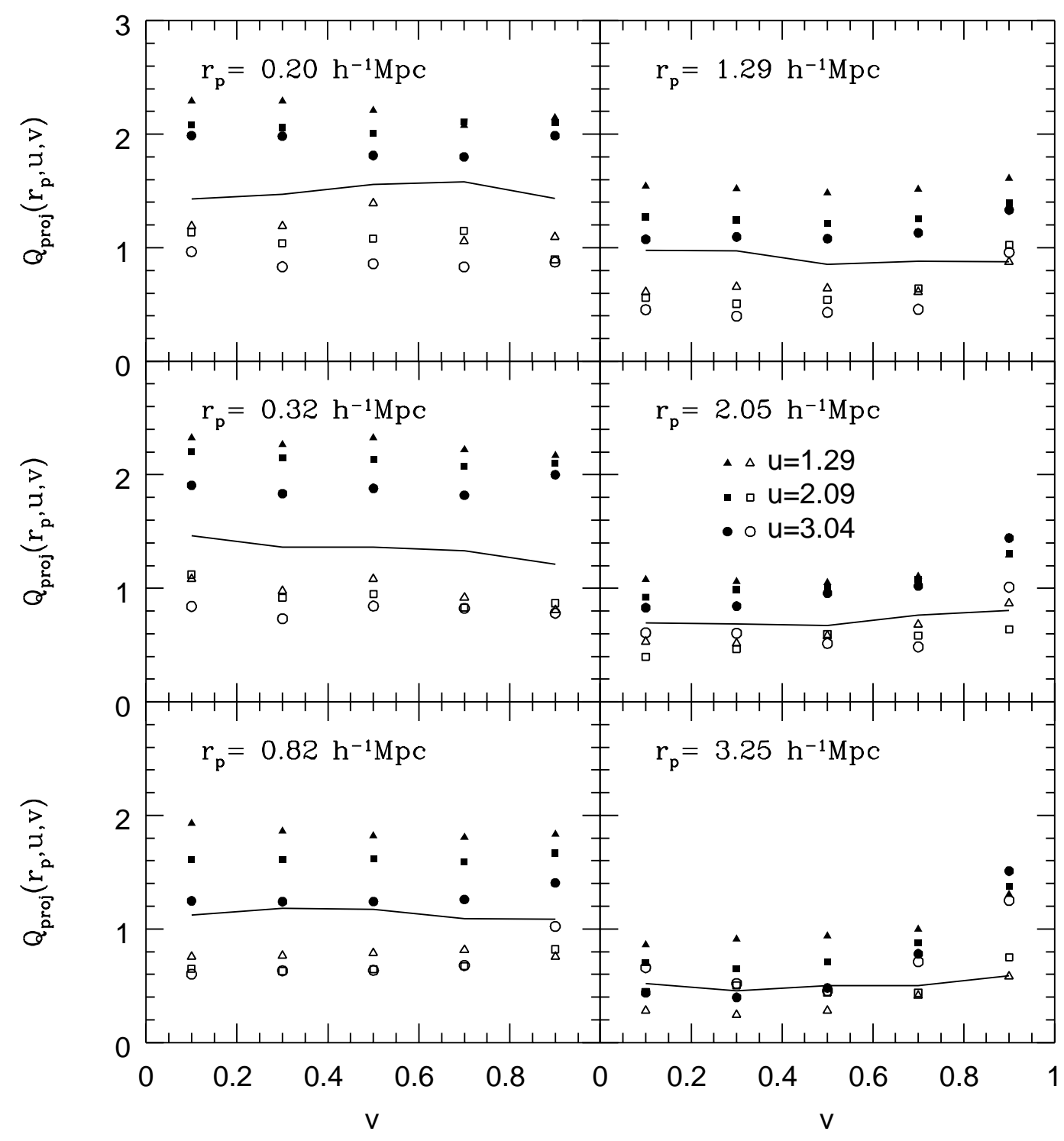

Fig. 6.- The normalized projected 3PCF of the LCRS survey (open symbols) compared with the mean value of the ten mock samples (solid symbols). The thick solid lines represent the lowest of the ten mock $Q_{r e d}(s, u, v)$ values in each $u=1.29$ bin, which can be regarded as the lower model limits at $\sim 90 \%$ significance level. 